\title{
Conditions for the Existence of Fixed Points in a Finite System of Kuramoto Oscillators
}

\author{
Mark Verwoerd \& Oliver Mason
}

\begin{abstract}
We present new necessary and sufficient conditions for the existence of fixed points in a finite system of coupled phase oscillators on a complete graph. We use these conditions to derive bounds on the critical coupling.
\end{abstract}

\section{INTRODUCTION}

Synchronized behaviour has been widely observed in natural and engineered systems [13], [2], [12], [5], and understanding the mechanisms behind its emergence is a key issue in the study of interconnected dynamical systems. For some time now, there has been considerable interest across the mathematics, physics and engineering communities in the development and analysis of simple mathematical models of synchronization [8], [14], [15], [3], [16], [17]. One of the most popular frameworks for the mathematical study of synchronization is the so-called Kuramoto model of phase coupled oscillators [9], [10], [18], [19], [1]. To date, several characteristics of this model have been determined. For instance, at very low values of the coupling strength, little or no synchronization is observed. As the coupling strength is increased, some partial synchronization appears in the network up to a threshold value of the coupling strength, referred to here as the critical coupling, at which fully synchronized behaviour emerges [8], [3].

The traditional Kuramoto model assumes that all pairs of oscillators in the network are connected with the same coupling strength [9]. This type of coupling is referred to as 'all-to-all' coupling and corresponds to a network in which the underlying graph is complete [4]. Other traditional classes of networks to have been considered include lattices [7] and rings [15]. More recently, the synchronization of coupled oscillators on networks with small-world [22], [21], [6] and scale-free [11] topologies has also attracted attention. Most recent results on synchronization and dynamics on networks have either been based on numerical simulations or else have been derived for the limiting case of networks of infinite size. To date, relatively few rigorous results are available for finite-size networks [18], [8], and there is a clear need for analysis techniques to gain insight into socalled finite-size effects. In this paper, we shall be concerned with synchronization in finite systems of coupled oscillators. Specifically: we shall establish (new) necessary and sufficient conditions for the existence of fixed points in a finite system of coupled oscillators (see also [20]) and compute bounds on the critical coupling strength for such systems. Our analysis

This work was not supported by any organization

M. Verwoerd and Oliver Mason are with the Hamilton Institute, National University of Ireland, Maynooth, Co. Kildare, Ireland \{mark. verwoerd, oliver. mason\} @nuim. ie is in the spirit of the work presented in [8], [3], specializing to the case of all-to-all coupling with particular emphasis on the existence of fixed points. The stability of such fixed points is also a topic of great interest, and has been considered in [8], [14], [15], [3], but shall not be addressed in the current paper.

The outline of the paper is as follows. In Section 2, we introduce the Kuramoto model, and review some of its basic properties. Here, we also give a formal definition of the critical coupling. In Section 3, we state the main results. In Section 4 a numerical example is presented. Section 5 closes with conclusions and an outlook on future research.

\section{THE KURAMOTO MODEL}

For the remainder of this paper, we shall be concerned with the Kuramoto model of coupled phase oscillators under the assumption of all-to-all coupling. Formally, this model is given by

$$
\dot{\theta}_{i}=\omega_{i}+\frac{k}{N} \sum_{j=1}^{N} \sin \left(\theta_{j}-\theta_{i}\right), \quad i=1, \ldots, N .
$$

Here, $\theta_{i}(\cdot) \in \mathbb{R}\left(S^{1}\right)$ and $\omega_{i} \in \mathbb{R}$ respectively denote the phase and intrinsic (or natural) frequency of oscillator $i$, and the constant $k \in \mathbb{R}_{+}$is a global coupling coefficient.

\section{A. The order parameter}

Let $\mathbb{D}$ denote the complex unit disc $\{z \in \mathbb{C}:|z| \leq 1\}$. We consider a map $r: \mathbb{R}^{N} \mapsto \mathbb{D}$, defined as follows:

$$
r(\theta):=\frac{1}{N} \sum_{j=1}^{N} e^{i \theta_{j}} .
$$

Let $r^{-1}(z):=\left\{\theta \in \mathbb{R}^{N}: r(\theta)=z\right\}$ denote the preimage of $r$, and note that the preimage is nonempty for all $z \in \mathbb{D}$ provided $N \geq 2$. We introduce the notation $\mathscr{R}_{0}:=r^{-1}(0)$. It shall be convenient to express $r(\theta)$ in terms of polar coordinates:

$$
r(\theta)=\left\{\begin{array}{ll}
R(\theta) e^{i \psi(\theta)} & \theta \in \mathbb{R}^{N} \backslash \mathscr{R}_{0} \\
0 & \theta \in \mathscr{R}_{0}
\end{array} .\right.
$$

Here, $R: \mathbb{R}^{N} \mapsto[0,1]$ and $\psi: \mathbb{R}^{N} \backslash \mathscr{R}_{0} \mapsto[0,2 \pi)$ are respectively defined as

$$
R(\theta):=\sqrt{\left(\frac{1}{N} \sum_{j=1}^{N} \sin \left(\theta_{j}\right)\right)^{2}+\left(\frac{1}{N} \sum_{j=1}^{N} \cos \left(\theta_{j}\right)\right)^{2}},
$$

and

$$
\psi(\theta):=\arctan \left(\frac{\frac{1}{N} \sum_{j=1}^{N} \sin \left(\theta_{j}\right)}{\frac{1}{N} \sum_{j=1}^{N} \cos \left(\theta_{j}\right)}\right) .
$$


It is not hard to see that the the magnitude $R(\theta)$ of $r(\theta)$ is invariant under transformations of the form $\theta \mapsto \theta+c \mathbf{1}$, where $\mathbf{1}$ denotes the vector of all ones and $c$ is a real number. In fact, this follows directly from Eqn. (2):

$$
R(\boldsymbol{\theta}+c \mathbf{1}):=\left|\frac{1}{N} \sum_{j=1}^{N} e^{i\left(\theta_{j}+c\right)}\right|=\left|e^{i c}\right| R(\boldsymbol{\theta})=R(\boldsymbol{\theta}) .
$$

By the same token, one can show that, for $\theta \in \mathbb{R}^{N} \backslash \mathscr{R}_{0}$,

$$
\psi(\theta+c \mathbf{1})=\psi(\theta)+c \bmod 2 \pi .
$$

In the physics literature, $r(\cdot)$ is known as the order parameter. $\left\{e^{i \theta_{j}}: j=1, \ldots, N\right\}$. Using definitions (4) and (5), we rewrite Eqn. (1), as follows:

$$
\dot{\theta}_{i}= \begin{cases}\omega_{i}+k R(\theta) \sin \left(\psi(\theta)-\theta_{i}\right) & \theta \in \mathbb{R}^{N} \backslash \mathscr{R}_{0}, \\ \omega_{i} & \theta \in \mathscr{R}_{0} .\end{cases}
$$

It follows from (3) that for $\theta \in \mathbb{R}^{N} \backslash \mathscr{R}_{0}$,

$$
R(\theta)=e^{-i \psi(\theta)} r(\theta)=\frac{1}{N} \sum_{j=1}^{N} e^{i\left(\theta_{j}-\psi(\theta)\right)} .
$$

Equating real and imaginary parts in (9), we obtain the following two identities for $\theta \in \mathbb{R}^{N} \backslash \mathscr{R}_{0}$ :

$$
\begin{gathered}
R(\theta)=\frac{1}{N} \sum_{j=1}^{N} \cos \left(\psi(\theta)-\theta_{j}\right) ; \\
\sum_{j=1}^{N} \sin \left(\psi(\theta)-\theta_{j}\right)=0 .
\end{gathered}
$$

\section{B. Fixed points}

Let $V \in \mathbb{R}^{N \times N}$ be given as

$$
\left[V_{i j}\right]:=\left\{\begin{array}{cc}
\frac{N-1}{N} & j=i \\
-\frac{1}{N} & j \neq i
\end{array} .\right.
$$

We define new coordinates $x(t):=V \theta(t)$. Note that $V$ is a projection matrix that maps $\mathbb{R}^{N}$ onto the $N-1$ dimensional linear subspace $V \mathbb{R}^{N}:=\left\{x \in \mathbb{R}^{N}: \sum_{j=1}^{N} x_{j}=0\right\}$. Define $\Omega:=$ $V \omega$. In the new coordinates, the system dynamics read:

$$
\dot{x}=\Omega+k f(x),
$$

where $f_{i}(x)$ is defined as:

$$
f_{i}(x):=\frac{1}{N} \sum_{j=1}^{N} \sin \left(x_{j}-x_{i}\right) .
$$

Or equivalently:

$$
f_{i}(x):=\left\{\begin{array}{ll}
R(x) \sin \left(\psi(x)-x_{i}\right) & x \in \mathbb{R}^{N} \backslash \mathscr{R}_{0} \\
0 & x \in \mathscr{R}_{0}
\end{array} .\right.
$$

The objective of this paper is to find conditions on $k$ and $\Omega$ under which the system (13) has one or more fixed points, where a fixed point is defined as follows.

Definition 1: Let $\Omega, x \in V \mathbb{R}^{N}$. We say that $x$ is a fixed point (of the system (13)) if $k f(x)=-\Omega$.

A fixed point in the sense of Definition 1 is a state of the system in which each oscillator is phase-locked to every other and moves at constant speed $\dot{\theta}_{i}=\langle\omega\rangle$. See also [8].

\section{Critical coupling}

Definition 2: Let $\Omega \in V \mathbb{R}^{N}$. The critical coupling, $k_{\mathrm{c}}$, is defined as:

$$
k_{\mathrm{c}}:=\inf _{k}\left\{k \in \mathbb{R}_{+}: \exists x \in V \mathbb{R}^{N} \text { s.t. } k f(x)=-\Omega\right\}
$$

We define the critical coupling $k_{\mathrm{c}}$ as the smallest $k$ for which the system (13) has at least one fixed point. Note that in the physics literature, the critical coupling has been defined alternatively as the smallest value of $k$ for which there exists at least one solution $x(t), t \geq t_{0}$, and a constant $c \in(0,1]$ such that $R(x(t))=c$ for all $t \geq t_{0}$ (so called stationary or steady solutions [18]). Our definition is identical to that of [8] (where the critical coupling is denoted as $K_{L}$ ).

\section{MAIN RESULTS}

\section{A. Lower bounds}

Inspection of Eqn. (13) shows that

$$
k_{\mathrm{c}} \geq\|\Omega\|_{\infty}:=\max _{i}\left|\omega_{i}-\langle\omega\rangle\right| .
$$

Eqn.(17) gives us a lower bound on the critical coupling. Using the next result, we shall derive another lower bound.

Theorem 1: Let $f(x)$ be given by (15). Then:

1) For all $x \in \mathbb{R}^{N}$,

$$
\|f(x)\|_{2} \leq \sqrt{N R^{2}(x)\left(1-R^{2}(x)\right)}
$$

2) If $N$ is even, then for every $c \in[0,1]$ there exists $x \in V \mathbb{R}^{N}$ such that $R(x)=c$ and $\|f(x)\|_{2}=$ $\sqrt{N R^{2}(x)\left(1-R^{2}(x)\right)}$

3) If $N$ is odd, then inequality (18) is strict for all $x \in \mathbb{R}^{N}$ such that $0<R(x)<1$.

Proof: Part 1. Observe that inequality (18) is trivially satisfied when $x \in \mathscr{R}_{0}$. Suppose therefore that $x \in \mathbb{R}^{N} \backslash \mathscr{R}_{0}$. Then by definition

$$
\begin{aligned}
\|f(x)\|_{2}^{2} & :=\sum_{j=1}^{N}\left(f_{j}(x)\right)^{2} \\
& =R^{2}(x) \sum_{j=1}^{N} \sin ^{2}\left(\psi(x)-x_{j}\right),
\end{aligned}
$$

where $\psi(x)$ and $R(x)$ are the phase and magnitude of the order parameter, previously defined in (5) and (4) respectively. Introducing the shorthand notation $z_{i}(x):=\cos \left(\psi(x)-x_{i}\right)$, and using (10) we now rewrite (19), as follows:

$$
\|f(x)\|_{2}^{2}=\left(\frac{1}{N} \sum_{j=1}^{N} z_{j}(x)\right)^{2} \sum_{j=1}^{N}\left(1-z_{j}(x)^{2}\right) .
$$

To derive the desired inequality we pick a $c \in[0,1]$ and maximize $\|f(x)\|_{2}$ over the set $\left\{x \in \mathbb{R}^{N}: R(x)=c\right\}$. We shall not solve this optimization problem directly, but take an indirect route by considering another, easier optimization problem, whose solution will then give us an upper bound on the solution to the first problem. Then we shall show that, under certain conditions, the two solutions coincide. To this end, let $c \in(0,1]$ and consider the constrained optimization problem 


OPT 1: \begin{tabular}{ll} 
maximize & $\sum_{j=1}^{N}\left(1-z_{j}(x)^{2}\right)$ \\
subject to & $\frac{1}{N} \sum_{j=1}^{N} z_{j}(x)=c, \quad x \in \mathbb{R}^{N} \backslash \mathscr{R}_{0}$ \\
\hline
\end{tabular}

Note that the constraint is feasible for all values of $c$ in the specified interval. We shall denote the solution to OPT 1 as $s_{1}(c)$. Next consider a second optimization problem,

OPT 2: $\begin{array}{ll}\text { maximize } & \sum_{j=1}^{N}\left(1-y_{j}^{2}\right) \\ \text { subject to } & \frac{1}{N} \sum_{j=1}^{N} y_{j}=c, \quad y \in \mathbb{R}^{N} .\end{array}$

and let the solution to this problem be denoted as $s_{2}(c)$. We then have that $s_{2}(c) \geq s_{1}(c)$ for all $c \in(0,1]$. In other words, the solution to OPT 1 is upper bounded by the solution to OPT 2. The solution to OPT 2 can be found by means of standard Lagrange multiplier techniques. The optimum $s_{2}(c)=N\left(1-c^{2}\right)$, is attained when $y_{i}=c$ for all $i$. We conclude that

$$
\max _{\left\{x \in \mathbb{R}^{N}: R(x)=c\right\}}\|f(x)\|_{2}^{2} \leq N c^{2}\left(1-c^{2}\right),
$$

and hence, $\|f(x)\|_{2} \leq \sqrt{N} R(x) \sqrt{1-R^{2}(x)}$. for all $x \in \mathbb{R}^{N}$. Part 2. To prove the second part of the theorem, let $c \in(0,1]$ and note that $s_{1}(c)=s_{2}(c)$ if and only there exists $x \in \mathbb{R}^{N} \backslash \mathscr{R}_{0}$ such that

$$
\cos \left(\psi(x)-x_{i}\right)=c
$$

for all $i$. Suppose $N$ is even and let $x$ be given as

$$
x_{i}:= \begin{cases}\arccos (c) & i=1, \ldots, \frac{N}{2} \\ -\arccos (c) & i=\frac{N}{2}, \ldots, N\end{cases}
$$

Then $\sum_{j=1}^{N} x_{j}=0$, and, by definition, $x \in V \mathbb{R}^{N}$. Moreover, $\psi(x)=0$, and $\cos \left(\psi(x)-x_{i}\right)=c$ for all $i$. This completes the second part. Part 3. To prove the third part, let $N$ be odd and suppose there exists $x \in \mathbb{R}^{N}$ such that Condition (22) is satisfied. Then it follows from the identity $\sin ^{2}\left(\psi(x)-x_{i}\right)+$ $\cos ^{2}\left(\psi(x)-x_{i}\right)=1$ that there must exist $a \in\{-1,1\}^{N}$ such that $\sin \left(\psi(x)-x_{i}\right)=a_{i} \sqrt{1-c^{2}}$ for all $i$. By Identity (11), we have that $\sum_{j} \sin \left(\psi(x)-x_{j}\right)=0$, which, assuming $c \neq 1$, implies that $\sum_{j=1}^{N} a_{j}=0$. But this cannot be true unless $N$ is even. Thus we arrive at a contradiction and we conclude that if $N$ is odd then $s_{2}(c)>s_{1}(c)$ for all $c$ such that $0<c<1$. This concludes the proof.

Figure 1 illustrates the result of Theorem 1. When $N=4$ (even), the lower bound is attained at every value of $R(x)$, which shows that the given bound is the tightest possible. However, as illustrated in the left panel, when $N=3$, the bound is never attained except on the subset of $\mathbb{R}^{3}$ defined by $\left\{x \in \mathbb{R}^{3}: R(x) \in\{0,1\}\right\}$.

Theorem 1 has a number of interesting implications. First of all it tells us something about the rate at which solutions converge to a fixed point. To see this, assume $\omega_{i}=\omega_{j}$ for all $(i, j)$ and consider the homogeneous system

$$
\left\{\begin{array}{ccc}
\dot{\theta}(t) & = & k f(\theta(t)) \\
\theta\left(t_{0}\right) & = & \theta_{0}
\end{array}\right.
$$

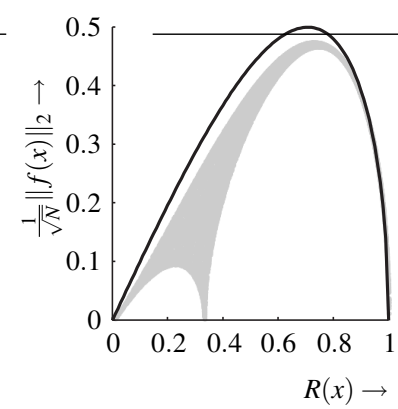

(a) $N=3$

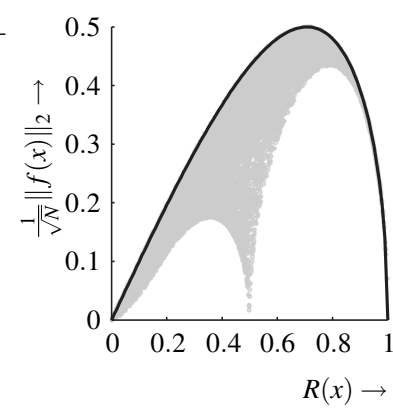

(b) $N=4$
Fig. 1. Scatter plot of $\frac{1}{\sqrt{N}}\|f(x)\|_{2}$ for $N=3$ (left panel) and $N=4$ (right panel). The phases $x$ were drawn from a uniform distribution. The solid black line in both panels is the upper bound $R(x) \sqrt{1-R^{2}(x)}$.

where $\theta_{0} \in \mathbb{R}^{N}$. The time derivative of the magnitude squared of the order parameter, $L(\cdot):=R^{2}(\cdot)$ is given as

$$
\frac{\mathrm{d} L(\theta(t))}{\mathrm{d} t}:=\frac{\partial L(\theta)}{\partial \theta} \dot{\theta}(t)=\frac{\partial L(\theta)}{\partial \theta} k f(\theta(t)) .
$$

Using the identity $\frac{\partial L(\theta)}{\partial \theta}=\frac{2}{N}[f(\theta)]^{T}$, it follows that

$$
\frac{\mathrm{d} L(\theta(t))}{\mathrm{d} t}=\frac{2 k}{N} \| f\left(\theta(t) \|_{2}^{2} .\right.
$$

The derivative is positive everywhere, except at the equilibria, where it is zero. It follows that the magnitude of the order parameter is a nondecreasing function of time. We formulate the following conjecture (see also [3], [8]):

Conjecture 1: For almost all initial conditions $\theta_{0}$, the solution $\theta(t)$ to the homogeneous system (24) has the property that $\lim _{t \rightarrow \infty} R(\theta(t))=1$.

In agreement with Conjecture 1, one can prove that, for the homogenous system, the global phase-locking manifold $\mathscr{M}:=\left\{\theta \in \mathbb{R}^{N}: \theta_{i}=\theta_{j}\right.$ for all $\left.i, j\right\}$ is (locally) asymptotically stable. However, the existence of other invariant manifolds, not contained in $\mathscr{M}$, implies that $\mathscr{M}$ is not globally asymptotically stable. We conjecture that $\mathscr{M}$ is 'almost globally asymptotically stable', in the sense that its region of attraction is the entire space minus a set of measure zero.

For our next result, we shall need the concept of a dominating function, which is defined as follows:

Definition 3: Let $f, g: \mathbb{R} \mapsto \mathbb{R}$ and let $\mathscr{I} \subset \mathbb{R}$ be an interval. We say that $f$ dominates $g$ on $\mathscr{I}$ if $f(t) \geq g(t)$ for all $t \in \mathscr{I}$. In that case we call $f$ a dominating function for $g$ on $\mathscr{I}$.

Using Theorem 1, we compute a dominating function for $L(\cdot)$, as follows.

Corollary 1: Let $\theta(\cdot)$ be a solution to the homogeneous system (24) with initial condition $\theta\left(t_{0}\right)=\theta_{0}$. Then

$$
D(t):=\frac{1}{1-e^{-2 k\left(t-t_{0}\right)}\left(\frac{L\left(\theta_{0}\right)-1}{L\left(\theta_{0}\right)}\right)}
$$

is a dominating function for $L(\theta(t))$ on $\left[t_{0}, \infty\right)$.

Proof: By (25) and Theorem 1 we have that $\dot{L}(\theta(t)) \leq$ $2 k L(\theta(t))(1-L(\theta(t)))$ for all $t$. This implies that, on $\left[t_{0}, \infty\right)$, 
$L(\theta(t))$ is dominated by the solution $y(t)$ of the ODE

$$
\left\{\begin{array}{ccc}
\dot{y} & = & 2 k y(1-y) \\
y\left(t_{0}\right) & = & L\left(\theta_{0}\right)
\end{array}\right.
$$

which is given as

$$
y(t)=\frac{1}{1-e^{-2 k\left(t-t_{0}\right)}\left(\frac{L\left(\theta_{0}\right)-1}{L\left(\theta_{0}\right)}\right)}, \quad t \geq t_{0} .
$$

This completes the proof.

Figure 2 shows the graph of $L(\theta(t))$ and that of the dominating function $D(t)$-Eqn. (26) for a particular realization of the initial condition $\theta_{0}$. In this example, $N=100$ and $k=2$. We observe that, as expected, the solution converges to the synchronized state, $L((\theta(t)) \rightarrow 1$.

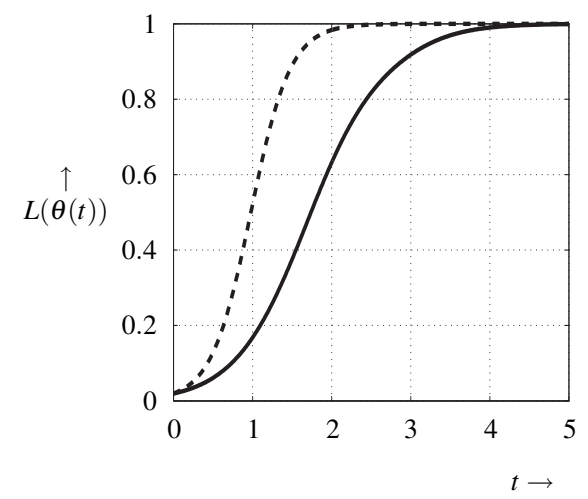

Fig. 2. Numerical simulation of the homogeneous system (24) with $N=100$ oscillators and coupling coefficient $K=2$ : time evolution of $L(t):=R^{2}(t)$ (solid line) and the dominating function $D(t)$-Eqn. (26) (dashed line).

Let $\sigma_{\omega}:=\sqrt{\frac{1}{N} \sum_{j=1}^{N}\left(\omega_{j}-\langle\omega\rangle\right)^{2}}$ denote the sample standard deviation of $\omega$. Using Theorem 1 we can derive a lower bound on the critical coupling, as follows:

Corollary 2: The critical coupling, $k_{\mathrm{c}}$, satisfies:

$$
k_{\mathrm{c}} \geq 2 \sigma_{\omega}
$$

Proof: Let $x^{*} \in V \mathbb{R}^{N}$ and suppose $x^{*}$ is a fixed point. Then by definition $k\left\|f\left(x^{*}\right)\right\|_{2}=\|V \omega\|_{2}=\sqrt{N} \sigma_{\omega}$ and by Theorem 1 we have that

$$
\left\|f\left(x^{*}\right)\right\|_{2} \leq \sqrt{N} \sqrt{R^{2}\left(x^{*}\right)\left(1-R^{2}\left(x^{*}\right)\right)}
$$

It is not hard to see that the right hand side of (30) is upper bounded by $\frac{1}{2} \sqrt{N}$. It follows that

$$
k_{\mathrm{c}} \geq \frac{\sqrt{N} \sigma_{\omega}}{\sqrt{N} \frac{1}{2}}=2 \sigma_{\omega}
$$

This completes the proof.

Note that the previous result is in agreement with the intuition that greater variation in intrinsic frequencies requires stronger coupling to achieve full synchronization.

\section{B. Necessary and Sufficient conditions}

Our next result provides a necessary and sufficient condition for the system (13) to have a fixed point, given a particular coupling strength $k$.

Theorem 2: Let $k>0$ and $\Omega \in V \mathbb{R}^{N}$. The system (13) has a fixed point iff there exists $\beta \in\left[\frac{1}{k}\|\Omega\|_{\infty}, 1\right] \subset \mathbb{R}$ and $a \in\{-1,1\}^{N}$ such that

$$
\beta=\frac{1}{N} \sum_{j=1}^{N} a_{j} \sqrt{1-\left(\frac{\Omega_{j}}{k \beta}\right)^{2}} .
$$

Proof: Suppose $\Omega \neq 0$ (the case $\Omega=0$ is easy). Let $x^{*} \in$ $V \mathbb{R}^{N}$ be a fixed point. By definition, $k f\left(x^{*}\right)=-\Omega$, and since $\Omega \neq 0$, we have that $f\left(x^{*}\right) \neq 0$, and consequently $R\left(x^{*}\right) \neq 0$. It follows that

$$
\sin \left(\psi\left(x^{*}\right)-x_{i}^{*}\right)=-\frac{\Omega_{i}}{k R\left(x^{*}\right)}, \quad i=1,2, \ldots, N .
$$

Let $\beta:=R\left(x^{*}\right)$. By (33) we have that $\beta \geq \frac{1}{k}\|\Omega\|_{\infty}$. Recall that for all $x \in \mathbb{R}^{N} \backslash \mathscr{R}_{0}, R(x)$ can be written as

$$
R(x)=\frac{1}{N} \sum_{j=1}^{N} \cos \left(\psi(x)-x_{j}\right),
$$

and let $a_{i}$ be given as

$$
a_{i}:= \begin{cases}-1 & \text { if } \cos \left(\psi\left(x^{*}\right)-x_{i}^{*}\right) \leq 0 \\ +1 & \text { otherwise }\end{cases}
$$

Combining (33), (34), and (35), we arrive at

$$
\beta=\frac{1}{N} \sum_{j=1}^{N} a_{j} \sqrt{1-\left(\frac{\Omega_{j}}{k \beta}\right)^{2}} .
$$

This proves necessity. To prove sufficiency, let $a \in\{-1,1\}^{N}$ be given, and suppose $\beta \geq \frac{1}{k}\|\Omega\|_{\infty}>0$ (again, the case $\Omega=0$ is easy). Then for every $c \in \mathbb{R}$, the system

$$
\left\{\begin{array}{l}
k \beta \sin \left(-y_{i}-c\right)=-\Omega_{i} \\
a_{i} \cos \left(-y_{i}-c\right) \geq 0,
\end{array} \quad i=1,2, \ldots, N\right.
$$

has a unique solution $y^{*} \in[-\pi, \pi)^{N}$. We pick $c$ such that $\sum_{j=1}^{N} y_{j}^{*}=0$. Since $\sum_{j=1}^{N} \sin \left(y_{j}^{*}+c\right)=0$, it follows that

$$
R\left(y^{*}\right)=R\left(y^{*}+c \mathbf{1}\right)=\left|\sum_{j=1}^{N} \cos \left(y_{j}^{*}+c\right)\right|
$$

From (37), we have that

$$
\cos \left(y_{i}^{*}+c\right)=a_{i} \sqrt{1-\left(\frac{\Omega_{i}}{k \beta}\right)^{2}} \quad i=1, \ldots, N .
$$

Combining (38) and (39), we arrive at

$$
R\left(y^{*}\right)=\left|\frac{1}{N} \sum_{j=1}^{N} a_{j} \sqrt{1-\left(\frac{\Omega_{j}}{k \beta}\right)^{2}}\right|
$$

Under the hypotheses of the theorem $\beta=R\left(y^{*}\right)$. It follows that $k R\left(y^{*}\right) \sin \left(\psi\left(y^{*}\right)-y_{i}^{*}\right)=-\Omega_{i}$, for $i=1,2, \ldots, N$. Hence, $y^{*}$ is a fixed point. This concludes the proof. 
Theorem 2 gives us a necessary and sufficient condition for the equation $k f(x)=-\Omega$ to have at least one solution for a given value of $k$. It is not clear, however, that there exists $k$ for which this condition is satisfied. The following Corollary provides an easy sufficient condition.

Corollary 3: Let $k>0$ and $\Omega \in V \mathbb{R}^{N}$. Suppose

$$
\frac{1}{k}\|\Omega\|_{\infty} \leq \frac{1}{N} \sum_{j=1}^{N} a_{j} \sqrt{1-\left(\frac{\Omega_{j}}{\|\Omega\|_{\infty}}\right)^{2}}
$$

for some $a \in\{-1,1\}^{N}$. Then the system (13) has at least one fixed point.

Proof: Suppose $\Omega \neq 0$ (again, the case $\Omega=0$ is easy). Let $a \in\{-1,1\}^{N}$. Define $g:\left[\frac{1}{k}\|\Omega\|_{\infty}, 1\right] \mapsto \mathbb{R}, g(\beta):=\beta$ and $H:\left[\frac{1}{k}\|\Omega\|_{\infty}, 1\right] \times\{-1,1\}^{N} \mapsto \mathbb{R}$,

$$
H(\beta, a):=\frac{1}{N} \sum_{j=1}^{N} a_{j} \sqrt{1-\left(\frac{\Omega_{j}}{k \beta}\right)^{2}} .
$$

Since $\Omega \neq 0$ we have that $g(1)>H(1, a)$. Now suppose condition (41) is satisfied. Then we have that $g\left(\frac{1}{k}\|\Omega\|_{\infty}\right) \leq$ $H\left(\frac{1}{k}\|\Omega\|_{\infty}, a\right)$, and by the Intermediate Value Theorem there must exist $\beta^{*} \in\left[\frac{1}{k}\|\Omega\|_{\infty}, 1\right]$ such that $g\left(\beta^{*}\right)=H\left(\beta^{*}, a\right)$. It follows from Theorem 2 that the system (13) has a fixed point.

Corollary 4: Let $k>0$ and $\Omega \in V \mathbb{R}^{N}$. Then the system (13) has at least one fixed point if and only if there exist $\beta \in\left[\frac{1}{k}\|\Omega\|_{\infty}, 1\right]$ such that

$$
\beta=\frac{1}{N} \sum_{j=1}^{N} \sqrt{1-\left(\frac{\Omega_{j}}{k \beta}\right)^{2}} .
$$

Proof: The proof of Corollary 3 suggests that if the fixed point equation (32) does not have a solution, then

$$
\beta>\frac{1}{N} \sum_{j=1}^{N} a_{j} \sqrt{1-\left(\frac{\Omega_{j}}{k \beta}\right)^{2}}
$$

for all $\beta \in\left[\frac{1}{k}\|\Omega\|_{\infty}, 1\right]$ and for all $a \in\{-1,1\}^{N}$. Since

$$
\frac{1}{N} \sum_{j=1}^{N} \sqrt{1-\left(\frac{\Omega_{j}}{k \beta}\right)^{2}} \geq \frac{1}{N} \sum_{j=1}^{N} a_{j} \sqrt{1-\left(\frac{\Omega_{j}}{k \beta}\right)^{2}}
$$

for all $a \in\{-1,1\}^{N}$, it follows that the given condition is necessary and sufficient for the system (13) to have at least one fixed point. This concludes the proof.

Our next and final corollary gives us an easy upper bound on the critical coupling.

Corollary 5: The critical coupling $k_{\mathrm{c}}$ satisfies

$$
k_{\mathrm{c}} \leq \frac{\|\Omega\|_{\infty}}{\frac{1}{N} \sum_{j=1}^{N} \sqrt{1-\left(\frac{\Omega_{j}}{\|\Omega\|_{\infty}}\right)^{2}} .}
$$

Proof: Follows directly from Corollary 3.

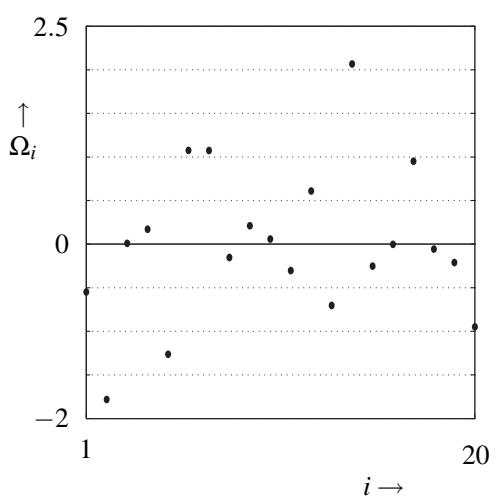

Fig. 3. The frequencies $\Omega_{i}:=\omega_{i}-\langle\omega\rangle$ used in the example.

\section{NumERICAL EXAMPLE}

We consider a system with $N=20$ oscillators, with 'natural frequencies' $\left\{\Omega_{i}\right\}$ as depicted in Figure 3.

For this example we have that $\|\Omega\|_{\infty}=2.07$ and

$$
\frac{1}{N} \sum_{j=1}^{N} \sqrt{1-\left(\frac{\Omega_{j}}{\|\Omega\|_{\infty}}\right)^{2}}=0.88
$$

It follows from Corollary 5 that $k_{\mathrm{c}} \leq 2.35$ and by (17), we have that $k_{\mathrm{c}} \geq\|\Omega\|_{\infty} \approx 2.07$. Figure 4 shows the time evolution of $R^{2}(t)$ (previously denoted as $L(t)$ ), for two different initial conditions and two values of the coupling coefficient, $k=2.3$ and $k=2.4$. We observe that in the second case, when $k$ is slightly greater than the known upper bound on $k_{\mathrm{c}}$, the value of $R^{2}(t)$ converges to a constant and inspection shows that the solution $x(t)$ of the system (13) tends to a fixed point. When $k=2.3$, convergence cannot be established. Note that in this case we do not know whether the system (13) has a fixed point or not, as the condition stated in Corollary 5 is only sufficient. To gain more insight into this case we fix the coupling coefficient at $k=2.3$, and numerically evaluate the function

$$
h(\beta, k):=\frac{1}{N} \sum_{j=1}^{N} \sqrt{1-\left(\frac{\Omega_{j}}{k \beta}\right)^{2}}
$$

for several values of $\beta$ in the interval $\left[\frac{1}{k}\|\Omega\|_{\infty}, 1\right]$. We repeat the same for $k=2.4$. The result is shown in Figure 5 . We observe that the equation $h(\beta, k)=\beta$ does not have a solution on the interval $\left[\frac{1}{k}\|\Omega\|_{\infty}, 1\right]$ when $k=2.3$, but does have a solution when $k=2.4$. By Corollary 4 , we have that $2.3 \leq k_{c} \leq 2.4$. Figure 5 suggests that the critical coupling corresponds to the smallest value of $k$ for which the graph of $h(\beta, k)$ intersects that of $g(\beta):=\beta$. It can be shown that, at the critical value of $k$, the point of intersection is unique (this follows from the fact that $h$ is a convex function and that, at this critical point, the partial derivative of $h$ with respect to $\beta$ must be equal to the derivative of $g$ with respect to $\beta$. From this it follows that the critical coupling can be found 


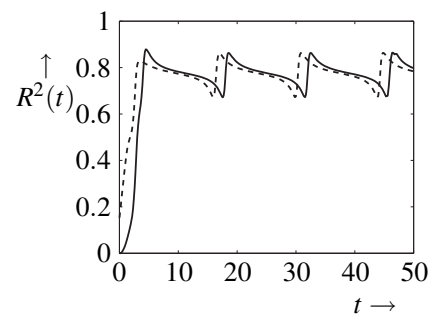

(a) $k=2.3$

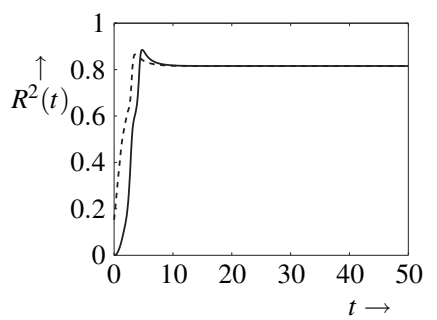

(b) $k=2.4$
Fig. 4. Time evolution of $R^{2}$ for two different initial condition (indicated by a dashed and solid line respectively), and two values of $k$. In the left panel, the value of $k(2.3)$ is slightly below the known upper bound on $k_{\mathrm{c}}$ and the system does not converge to a fixed point; in the right panel the value of $k(2.4)$ is slightly above the known upper bound on $k_{\mathrm{c}}$ and the system converges to a fixed point as expected.

numerically by determining the unique solution $u^{*}$ of

$$
\frac{1}{N} \sum_{j=1}^{N} \sqrt{1-\left(\frac{\Omega_{j}}{u}\right)^{2}}=\frac{1}{N} \sum_{j=1}^{N} \frac{\left(\frac{\Omega_{j}}{u}\right)^{2}}{\sqrt{1-\left(\frac{\Omega_{j}}{u}\right)^{2}}} .
$$

Indeed, let $u^{*}$ be the unique solution of (45) on $\left(\|\Omega\|_{\infty}, \infty\right)$. Then the critical coupling is given as

$$
k_{c}:=\frac{u^{*}}{\frac{1}{N} \sum_{j=1}^{N} \sqrt{1-\left(\frac{\Omega_{j}}{u^{*}}\right)^{2}}} .
$$

We remark that the solution $u^{*}$ of (45) can be found very efficiently by means of a bisection algorithm, noting that, on $\left(\|\Omega\|_{\infty}, \infty\right)$, the left hand side of (45) is a strictly increasing function of $u$, and the right hand side of (45) is a strictly decreasing function of $u$.

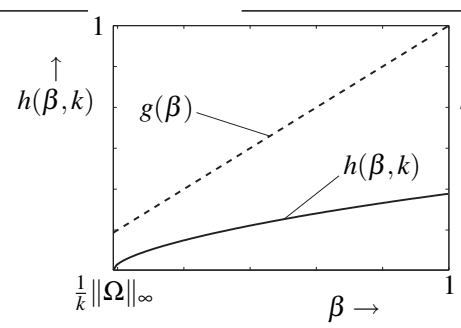

(a) $k=2.3$

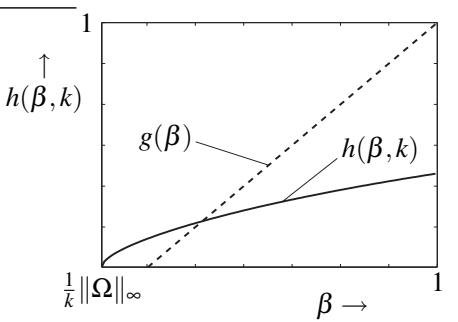

(b) $k=2.4$
Fig. 5. $h(\beta, k)$-Eqn. (44) vs. $\beta$ for $k=2.3,2.4$ and $\beta \in\left[\frac{1}{k}\|\Omega\|_{\infty}, 1\right]$. The dashed line is the graph of $g(\beta):=\beta$. An intersection corresponds to a solution of the fixed point equation $h(\beta, k)=g(\beta)$.

\section{CONCLUSION}

We derived necessary and sufficient conditions for the existence of fixed points in a finite system of coupled oscillators. In particular, we derived an easy sufficient condition in terms of the individual oscillator frequencies (Corollary 3), which we used to compute an upper bound on the critical coupling (Corollary 5). Simulation results indicate that this bound is tight, but at present we do not have analytical results to support this. In future work we shall seek to extend our analysis to complex networks of arbitrary topology, and investigate more closely the impact of the shape of the distribution on the value of the critical coupling.

\section{REFERENCES}

[1] J. Acebrón, L. Bonilla, C. Pérez Vicente, F. Ritort, and R. Spigler. The kuramoto model: a simple paradigm for synchronization phenomena. Reviews of Modern Physics, 77:137-185, 2005.

[2] A. Beuter, L. Glass, M.C. Mackey, and M.S. Titcombe, editors Nonlinear Dynamics in Physiology and Medicine. Springer, 2003.

[3] N. Chopra and M. Spong. On synchronization of kuramoto oscillators. In Proceedings of the 44th IEEE Conference on Decision and Control, Seville, Spain, pages 3916-3922, 2005.

[4] R. Diestel. Graph Theory. Springer-Verlag, 2000.

[5] L. Glass. Synchronization and rhythmic processes in physiology. Nature Review, 410:277-284, 2001.

[6] H. Hong, M. Choi, and B. Kim. Synchronization on small-world networks. Physical Review E, 65(026139), 2002.

[7] H. Hong, H. Park, and M. Choi. Collective synchronization in spatially extended systems of coupled oscillators with random frequencies. Physical Review E, 72(036217), 2005.

[8] A. Jadbabaie, N. Motee, and M. Barahona. On the stability of the kuramoto model of coupled nonlinear oscillators. In Proceedings of the American Control Conference, Boston, USA, pages 4296 - 4301, 2004.

[9] Y. Kuramoto. Self-entrainment of a population of coupled nonlinear oscillators. In H. Araki, editor, International Symposium on Mathematical Problems in Theoretical Physics, volume 39 of Lecture Notes in Physics. Springer, 1975.

[10] Y. Kuramoto. Chemical Oscillations, Waves and Turbulence. Springer, 1984.

[11] Y. Moreno and A. Pacheco. Synchronization of kuramoto oscillators in scale-free networks. Europhysics Letters, 68:603-609, 2004.

[12] J.D. Murray. Mathematical Biology. Springer, 3rd edition, 2002.

[13] A. Pikovsky, M. Rosenblum, and J. Kurths. Synchronization: a universal concept in nonlinear sciences. Cambridge University Press, 2003.

[14] J. Rogge and D. Aeyels. Existence of partial entrainment and stability of phase locking behavior of coupled oscillators. Progress of Theoretical Physics, 112:921-942, 2004.

[15] J. Rogge and D. Aeyels. Stability of phase locking in a ring of unidirectionally coupled oscillators. Journal of Physics A, 37:1113511148,2004

[16] R. Sepulchre. Oscillators as systems and synchrony as a design principle. In L. Menini, L. Zaccarian, and C. T. Abdallah, editors, Current trends in nonlinear systems and control, Lecture Notes in Control and Information Sciences. Birkhauser, 2006.

[17] R. Sepulchre, D. Paley, and N.E. Leonard. Group coordination and cooperative control of steered particles in the plane. In K. Y. Pettersen, J. T. Gravdahl, and H. Nijmeijer, editors, Group Coordination and Cooperative control, Lecture Notes in Control and Information Sciences, pages 217-232. Spinger-Verlag, London, 2006.

[18] S. Strogatz. From kuramoto to crawford: exploring the onset of synchronization in populations of coupled oscillators. Physica D, 143:1-20, 2000.

[19] S. Strogatz. Exploring complex networks. Nature, 410:268 - 276, 2001.

[20] M. Verwoerd. Fixed-point analysis of a finite system of kuramoto oscillators. In Proceedings of the Fourth Irish Conference on the Mathematical Foundations of Computer Science and Information Technology (MFCSIT), Cork, Ireland, 2006.

[21] D. Watts. Small Worlds: The Dynamics of Networks between Order and Randomness. Princeton University Press, 2003.

[22] D. Watts and S. Strogatz. Collective dynamics of 'small-world' networks. Nature, 393(6684)(6684):440-2, 1998. 